\title{
Quasi-diffraction limited emission from an array of tapered laser diodes in volume Bragg grating external cavities
}

\author{
David Paboeuf ${ }^{1}$, Gaëlle Lucas-Leclin ${ }^{1}$, Nicolas Michel ${ }^{2}$, Michel Calligaro ${ }^{2}$, Michel Krakowski ${ }^{2}$, \\ Patrick Georges ${ }^{1}$ \\ 1. Laboratoire Charles Fabry de l'Institut d'Optique, CNRS, Univ Paris-Sud, \\ Campus Polytechnique, RD128, 91127 Palaiseau Cedex, France \\ 2. Alcatel-Thales III-V Lab, RD128, 91767 Palaiseau - France
}

High-brightness single laser diodes based on the widespread taper design have demonstrated output powers of a few Watts with a single transverse mode operation [1]. The use of arrays of such lasers result in a further increase of the laser power, but with the drawback of a loss in the spatial brightness. To overcome this limitation numerous external-cavity configurations have been proposed which induce a coherence between the individual emitters of the array and result in a brightness improvement [2]. In this work we describe two external cavities intended to improve the spatial brightness of a bar of $\mathrm{N}=6$ index-guided tapered laser diodes emitting around $975 \mathrm{~nm}$. The lateral structure of the emitters consists of a short ridge single-mode section, a $2.3 \mathrm{~mm}$-long narrow-angle tapered ridge and a common amplified free-space $0.2 \mathrm{~mm}$-long section. The array pitch is $\mathrm{p}=30$ $\mu \mathrm{m}$, and the near-field $1 / \mathrm{e}^{2}$ full-width $\left(1 / \mathrm{e}^{2}-\mathrm{FW}\right)$ of each emitter is $30 \mu \mathrm{m}$ too, so the filling factor of this array is $100 \%$ on the front facet and the emission section is $\mathrm{w}=180 \mu \mathrm{m}$ wide. No adjacent coupling between emitters is evidenced in the free running laser emission of the array alone, and its $1 / \mathrm{e}^{2}-\mathrm{FW}$ divergence is $\sim 80 \mathrm{mrad}$ in the slow axis. Our external cavity designs aim at controlling the slow-axis beam divergence of the whole array by inducing an angular-filtered feedback into the lasers $[3,4]$. The configuration forces the array to operate in the out-of-phase mode, which has two main lobes in its far-field profile at $\pm \lambda / 2 p= \pm 16 \mathrm{mrad}$. We take benefit of the angular selectivity of volume Bragg gratings to favour an asymmetrical feedback on one of these peaks. The far-field of the extended-cavity array is thus expected to exhibit one diffraction-limited peak in the symmetric direction (Figure 1). Two different setups have been investigated experimentally:

In the design $\mathrm{T}$ (figure 1), a transmission Bragg grating with a diffraction efficiency of $90 \%$ and a full-width at half-maximum (FWHM) angular selectivity of $9 \mathrm{mrad}$ is inserted in the external cavity. A high reflection dielectric mirror reflects the diffracted beam back into the emitters. We observed a narrow 6 mrad-FWHM peak in the slow-axis far-field profile, which contains $30 \%$ of the output power. The peak width is close to the diffraction limit $\lambda / \mathrm{w}=5 \mathrm{mrad}$, and its $\mathrm{M}^{2}$ parameter is $<2$. The maximum output power reaches $1.3 \mathrm{~W}$ at $3 \mathrm{~A}$.

In the design $R$ (figure 1) a reflection Bragg grating with a reflectivity $R \geq 99 \%$ at $\lambda_{\mathrm{B}}=979 \mathrm{~nm}$ and a spectral bandwidth $\delta \lambda \cong 0.3 \mathrm{~nm}$ reflects the output beam at the angle of $+\lambda / 2 \mathrm{p}$. The angular selectivity of the grating is about $\Delta \theta_{\mathrm{R}}=35 \mathrm{mrad}$. A narrow peak appears in the far-field at $-\lambda / 2 \mathrm{p}$ which contains up to $50 \%$ of the total output power (figure 2), with a $\mathrm{M}^{2}$ parameter $<2$. Furthermore the laser spectrum is locked to the Bragg wavelength $\lambda_{B}$ resulting in a $<0.1 \mathrm{~nm}$-wide stabilized line. The total output power reaches $700 \mathrm{~mW}$ at $3 \mathrm{~A}$.

These two configurations both result in quasi-diffraction limited far-field profiles and similar output powers in the main lobe; nevertheless the setup $\mathrm{R}$ allows concurrent spectrum stabilization.

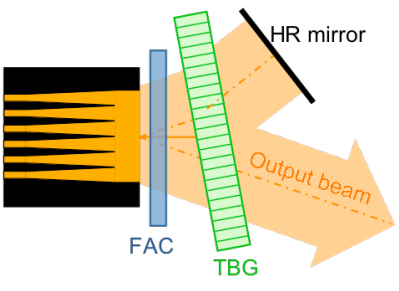

$\underline{\text { Setup T }}$

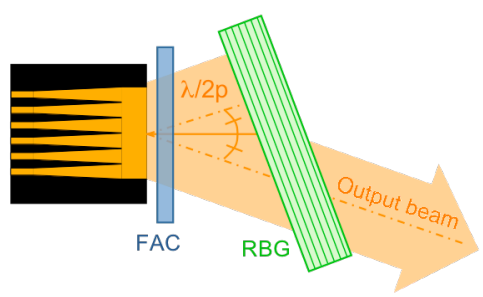

Setup R

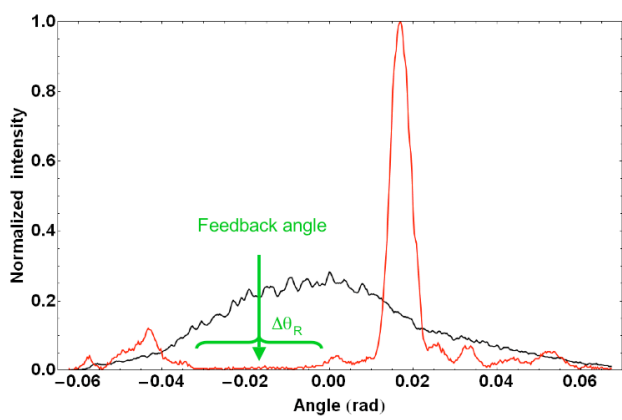

Fig. 1 Experimental external cavity setups; FAC = fast-axis collimation lens, $\mathrm{TBG}=$ transmission Bragg grating, $\mathrm{RBG}=$ reflection Bragg grating.

Fig. 2 Far-field profile of the free-running array (black) and of the external-cavity array in the R setup (red) at $\mathrm{I}=2.4 \mathrm{~A}$.

\section{Acknowledgments}

The authors thank the European Community for financial support under the www.BRIGHTER.eu program (IP 035266). D. Paboeuf acknowledges the funding of his $\mathrm{PhD}$ by the French Délégation Générale de l'Armement.

\section{References}

[1] H. Wenzel, B. Sumpf, G. Erbert, « High-brightness diode lasers », C. R. Physique 4, 649 (2003)

[2] D. Paboeuf, G. Lucas-Leclin, P. Georges, N. Michel, M. Krakowski, J. Lim, S. Sujecki, and E. Larkins, "Narrow-line coherently combined tapered laser diodes in a Talbot external cavity with a volume Bragg grating", App. Phys. Lett. 93, 211102 (2008)

[3] C. J. Chang-Hasnain, J. Berger, D. R. Scifres, W. Streifer, J. R. Whinnery, and A. Dienes, "High power with high efficiency in a narrow single-lobed beam from a diode laser array in an external cavity", App. Phys. Lett. 50, 1465 (1987)

[4] A. Barthelemy, F. Louradour and V. Courderc, "Wavelength-tunable diffraction-limited operation of a standard high-power diode-laser array using an off-centered extended cavity", Elec. Lett. 28, 2038 (1992) 